Titre : Hésitation face à la vaccination : considérations éthiques vues de multiples perspectives

Auteur : Kieran C. O'Doherty, Christine Smith et C. Meghan McMurtry

Publication : La santé publique à une ère marquée par le doute - Origines religieuses et culturelles de l'hésitation des Canadiens face à la vaccination

Directeurs : Paul Bramadat, Maryse Guay, Julie A. Bettinger et Réal Roy

Pages : $51-72$

ISBN : 978-2-7622-0359-2

URI : http://hdl.handle.net/11143/16025

DOI : https://doi.org/10.17118/11143/16025

Éditeur : Les Éditions de l'Université de Sherbrooke (ÉDUS) 


\title{
Hésitation face à la vaccination :considérations éthiques vues de multiples perspectives
}

\author{
KIERAN C. O'DOHERTY, CHRISTINE SMITH ET C. MEGHAN MCMURTRY
}

\section{Introduction}

La réticence et l'opposition à la vaccination sont associées à des enjeux éthiques difficiles. Lorsque, par exemple, une personne refuse de se faire vacciner, peut-on l'obliger à le faire pour sa propre protection et pour celle de la collectivité? Est-il possible de vacciner des enfants contre la volonté de leurs parents? À quel compromis peut-on en arriver entre la liberté de religion et les enjeux de santé publique, quand un groupe refuse la vaccination pour des motifs religieux? Ce sont des questions épineuses, car les personnes comme les collectivités peuvent y répondre en mettant l'accent sur des valeurs différentes.

Au moment d'analyser les répercussions de la réticence et de l'opposition à la vaccination sur le plan de l'éthique, le point de départ réside dans une bonne compréhension de la démarche des personnes et des groupes qui décident d'accepter ou de refuser des vaccins recommandés. Un problème se pose, car la documentation scientifique fait état d'importantes mésententes sur la manière d'y arriver. En fait, les disciplines proposent des points de vue différents pour nous aider à distinguer les motifs des décisions que prennent les citoyens. Nous démontrons, dans le présent chapitre, que des enjeux éthiques apparaissent ou sont dissimulés selon l'approche disciplinaire globale choisie. Nous nous concentrerons sur les quatre perspectives suivantes, qui se distinguent assez bien les unes des autres: 
1. La perspective de santé publique. Cette perspective se rapporte au mandat de protection de la santé à l'échelle de la société, parfois contraire à celle de la santé individuelle.

2. La perspective de la communication du risque. Cette perspective met la personne en vedette; on suppose que les décisions en matière de vaccination comportent un traitement cognitif des risques et des bienfaits.

3. La perspective psychologique de la santé. Cette perspective met aussi la personne en vedette, mais va au-delà de l'insistance sur l'information au sujet du risque; elle comporte un examen des facteurs psychologiques généraux pouvant influencer les décisions en matière de santé.

4. La perspective socioculturelle. Cette perspective prend en considération les facteurs politiques et historiques entrant en jeu dans l'adoption de la vaccination; on analyse les décisions individuelles d'acceptation ou de refus d'un vaccin en les considérant comme imbriquées dans un contexte global, à la fois social, religieux et culturel.

Dans ce chapitre, nous examinons la réticence et l'opposition à la vaccination selon chacune de ces perspectives et nous indiquons comment, en fonction de chacune, les décisions personnelles sont conceptualisées et comment cela entraîne une interprétation donnée ou la mise en vedette de certains enjeux de nature éthique. Quoique persuadées que chacune de ces perspectives permet de mieux comprendre les phénomènes étudiés par les auteurs du présent ouvrage, nous avons pour opinion générale qu'il y a beaucoup à gagner en adoptant le plus large point de vue possible, sur le plan socioculturel et politique, au moment de considérer ces enjeux éthiques. En outre, quoique nous présentions quatre perspectives distinctes, nous reconnaissons qu'un examen plus serré y fera apparaître bien des chevauchements. En guise de conclusion, nous partagerons quelques réflexions sur la valeur pratique de telles considérations.

\section{La perspective de santé publique}

Les vaccins sont réputés être une des plus grandes réalisations dans la lutte contre les maladies contagieuses et la promotion de la santé humaine (Ulmer et coll., 2006). Dans la perspective de santé publique, les programmes de vaccination représentent une des plus puissantes interventions disponibles pour protéger la santé des personnes et des populations. L'immunité collective - c'est-à-dire la protection indirecte offerte à tous les membres d'une société grâce à une couverture vaccinale élevée - figure parmi les volets importants de ces programmes. Comme beaucoup de non-vaccinés sont indirectement protégés de l'infection parce qu'ils rencontreront probablement moins d'organismes infectieux en présence de taux de vaccination globale élevés, on peut accepter sans malaise les demandes d'exemption des personnes pour qui un vaccin est contre-indiqué ou dont la réponse immunitaire est insatisfaisante. Des problèmes surgissent toutefois lorsque les taux de vaccination déclinent en deçà des niveaux nécessaires à l'immunité collective (généralement fixé à environ 90 à 95 p. cent, selon la maladie). Comme l'expliquent Roy, Bettinger et MacDonald (au quatrième et au huitième chapitres du présent ouvrage), des situations de ce genre se sont produites dans de nombreux pays, pour différentes maladies auparavant bien contrôlées. Par ailleurs, selon Bramadat (chapitre 1 du présent ouvrage) et Brunk (chapitre 3), on se préoccupe de plus en plus du fait que nombre de gens refusent la vaccination pour des motifs religieux, culturels ou idéologiques plutôt que médicaux. 
Dans une perspective de santé publique, le déclin de la couverture vaccinale représente une certaine énigme. Les données empiriques montrent l'importance d'une participation élevée aux programmes de vaccination. En règle générale, dans la perspective des responsables de santé publique, il n'y a pas de doute que des taux de vaccination élevés sont avantageux autant pour les personnes que pour l'ensemble de la population. Cependant, sauf en de rares circonstances, la participation ne peut être obligatoire, parce que la société s'est engagée à respecter l'autonomie individuelle et les principes démocratiques'. En termes de santé publique, le problème s'exprime donc par une question : comment peut-on encourager les populations et les personnes à adhérer volontairement aux programmes de vaccination? La volonté de promouvoir une couverture vaccinale élevée s'est souvent traduite, de la part des autorités en santé publique, par l'offre d'incitatifs (en Australie, prestations de maternité et allocations universelles pour les soins aux enfants sur présentation d'une preuve de vaccination; Salmon et coll., 2006) et l'imposition de moyens de dissuasion (p. ex., obstacles à l'inscription scolaire pour les personnes demandant une exemption de la vaccination; Siegal, Siegal et Bonnie, 2009).

Un des exemples les plus compliqués des défis que doivent relever les autorités de santé publique (et qui sont directement liés à la perspective dont nous traitons maintenant) se trouve dans la question de la vaccination des travailleurs de la santé. Dans de nombreux pays, ces travailleurs doivent êtr e vaccinés contre plusieurs maladies. On applique de telles politiques parce qu'ils sont soumis à un risque accru de contracter et de transmettre des maladies pouvant être prévenues par un vaccin en raison de leur contact direct avec des patients contagieux ou du matériel infecté (CDC, 2011). La vaccination obligatoire, dans leur cas, contribue à l'accomplissement de leur devoir d'éviter de nuire à autrui, car des moyens sont pris pour prévenir la transmission d'une maladie évitable (Anikeeva, Braunack-Mayer et Rogers, 2009). Elle favorise également la sécurité des travailleurs, en les protégeant contre les maladies contagieuses dans leur milieu de travail. Cependant, par sa définition même, la vaccination obligatoire diminue le libre arbitre des travailleurs de la santé et il faut, au moment de décider quel vaccin est nécessaire et quel autre ne l'est pas, apporter un soin particulier pour équilibrer des principes éthiques conflictuels. Depuis quelques années, nous avons observé maintes situations où des travailleurs de la santé n'ont pas respecté des programmes de vaccination de leur gouvernement ou de leur employeur.

Par ailleurs, les exemptions de la vaccination pour motifs culturels ou religieux sont généralement accordées, ce qui soulève un problème conceptuel dans la perspective de santé publique. Prenons l'exemple de patients ou même de travailleurs de la santé, appartenant à de petits groupes religieux et qui refusent de se faire vacciner en se fondant sur leurs croyances ou leur doctrine religieuses et en plaidant qu'elles ont préséance sur les considérations de santé individuelle ou collective. Dans ce cas-là, on ne peut pas nécessairement dire que ces gens rejettent les arguments en faveur de l'efficacité de la vaccination, l'information sur les risques propres aux maladies et les autres messages de santé publique. C'est plutôt que ces personnes jugent de telles questions non pertinentes ou négligeables, par rapport à leur fidélité à une doctrine donnée (citons le cas, il y a de nombreuses années, de catholiques rendus mal à l'aise par l'utilisation de lignées cellulaires de fœetus avortés pour la production de vaccins) ou à une cosmologie (celle du mouvement de la Science chrétienne, par exemple,

1. Il est important de reconnaître que les services de santé publique ont le pouvoir d'utiliser des mesures plus coercitives dans des circonstances extrêmes, par exemple en cas d'épidémie ou de pandémie grave. 
où l'on s'interroge sur la nécessité des vaccins puisque la prière est si puissante et que la maladie est irréelle). Comme ce mode de résistance n'est pas fondé sur une mésentente quant à la nature ou l'ampleur des risques pour la santé, il est fort peu probable qu'on obtienne le résultat voulu en inondant les réticents de données empiriques portant sur les risques de la vaccination (par rapport à ceux qu'entraîne la maladie ou d'autres conditions indésirables, par exemple). Les autorités de santé publique semblent alors n'avoir d'autre recours, pour accomplir leur mandat de promouvoir la santé, que de rendre la vaccination obligatoire et d'obliger les personnes et les groupes à l'accepter contre leur gré.

Lorsque la couverture vaccinale d'une population est élevée et que l'immunité collective est obtenue, les personnes non vaccinées sont aussi protégées, quoique indirectement. Les personnes qui choisissent de se faire vacciner prennent donc le risque de subir les effets indésirables du vaccin tout en procurant aux autres les avantages de la protection indirecte. On juge cette situation généralement justifiée sur le plan éthique si les personnes non vaccinées arborent une condition médicale (comme une allergie à l'un des composants d'un vaccin) qui leur interdit la vaccination. En revanche, quand la décision de refuser un vaccin n'est pas basée sur un motif de santé, il s'ensuit une controverse fondée sur la justice et l'équité. Somme toute, dans une situation d'immunité collective, les personnes qui refusent la vaccination profitent de l'immunisation fournie par le vaccin sans avoir à en payer le " coût », qu'il soit financier, émotionnel (peur du vaccin lui-même ou de la douleur causée par l'aiguille) ou médical (risque d'une réaction indésirable)². On appelle parfois ces personnes des « resquilleurs »et certains prétendent qu'il est contraire à l'éthique de refuser un vaccin en l'absence d'un motif légitime (Isaacs et coll., 2009), encore qu'on ne s'entende pas sur ce qui est légitime et ce qui ne l'est pas. Le problème se complique quand le nombre des resquilleurs est si grand que la couverture vaccinale chute sous le seuil critique et entraîne la perte de l'immunité collective. Quand cela se produit, n'ayant de protection ni directe, ni indirecte, ils deviennent un groupe vulnérable. Une décision de refus de la vaccination peut donc être considérée comme contraire à l'éthique sur la base d'arguments comme la justice, la solidarité et la santé publique, puisqu'un grand nombre de personnes non vaccinées mettent en danger des vies en créant une vulnérabilité à la maladie dans la population. Outre le risque que ces personnes s'imposent à elles-mêmes et aux autres non-vaccinés, il faut tenir compte d'un autre problème critique, à savoir l'élévation du risque pour les nourrissons trop jeunes et les personnes trop malades pour être vaccinés. De fait, dans la perspective de santé publique, les arguments en faveur de la vaccination obligatoire peuvent s'appuyer sur l'éthique quand on considère à la fois l'équité (tous doivent supporter également le fardeau de la vaccination, pour que tous puissent en profiter) et du bien-être collectif (pour atteindre et conserver une protection indirecte bénéficiant aux personnes les plus vulnérables de la population qu'il est impossible de protéger directement).

Une autre complication d'ordre éthique surgit lorsque les parents refusent la vaccination pour leurs enfants. Comme les jeunes enfants ne sont généralement pas considérés comme étant suffisamment compétents pour prendre des décisions par eux-mêmes, on se demande évidemment qui, de l'État ou de leurs parents, est le mieux placé pour défendre leurs intérêts. On attribue aux parents la responsabilité morale de prendre soin de leurs enfants et nombreux sont ceux qui conviennent qu'ils ont le droit de le faire comme le prescrivent leurs croyances

2. Nous incluons ici : 1) les cas particuliers de personnes qui ne peuvent être immunisées à cause d'une immunosuppression ou d'une allergie aux œufs et 2) les personnes qui manifestent une réaction indésirable globale aux vaccins, d'envergure légère (douleur au bras) ou grave. 
en matière de culture et de religion, à moins que celles-ci ne causent du tort à l'enfant (Isaacs et coll., 2009). L'autonomie des parents, à cet égard, serait cependant restreinte lorsque la vaccination est obligatoire pour les enfants ou lorsque le refus est associé à de fortes mesures de dissuasion (Field et Caplan, 2008; Salmon et coll., 2006). Par conséquent, en considérant les enjeux éthiques clés, il serait bon de se demander à quel point et dans quelles circonstances l'État est justifié d'intervenir pour assurer le bien-être d'un enfant jugé à risque parce que ses parents ont décidé de ne pas le faire vacciner (Asser et Swan, 1998). II va sans dire que la réponse varie en fonction des valeurs sociales et de l'encadrement normatif souvent propres à l'époque et au lieu visés. Autrement dit, en cas d'éclosion importante de rougeole (maladie peu grave pour certains, mais pouvant avoir des répercussions importantes), on peut s'attendre à ce que la société tolère plus facilement (en général) l'imposition de la vaccination obligatoire par l'État. Selon les politiques actuelles, les autorités de santé publique ont toutefois pour tâche de protéger la santé de la population. Leur personnel, dont le mandat, voire l'impératif éthique, consiste à protéger les gens vulnérables, doit donc s'intéresser particulièrement aux enfants qui sont soumis à plus de risque à cause de la décision de refuser la vaccination prise par leurs parents.

Sur cette base, nous pouvons dire que, dans une perspective de santé publique, les décisions d'opposition à la vaccination sont de quatre types, c'est-à-dire justifiées, erronées, contraires à l'éthique ou non pertinentes.

1. Décisions justifiées. Comme la vaccination peut entraîner des réactions indésirables, certains exigent que les programmes connexes répondent aux plus hautes normes d'efficacité et de sécurité (Schwartz et Caplan, 2011; Verweij et Dawson, 2004). Quand on a toutes les raisons de croire qu'une personne est plus vulnérable qu'une autre aux réactions graves associées à un vaccin, la décision de ne pas la vacciner est justifiée.

2. Décisions erronées. Quand on se rend compte que des gens prennent des décisions sur la base de renseignements incorrects, celles-ci peuvent sembler erronées, voire irrationnelles. D'autres auteurs, dans le présent ouvrage, ont indiqué qu'il est possible de surestimer le risque de réactions indésirables et de sous-estimer autant la gravité de la maladie que la vulnérabilité à celle-ci.

3. Décisions contraires à l'éthique. On peut dire qu'une décision de refus de la vaccination est contraire à l'éthique quand elle met des enfants en danger. C'est le cas également des resquilleurs, qui profitent des avantages de la vaccination sans en payer le coût et qui dès lors mettent en péril des populations entières et particulièrement les personnes qui ne sont pas protégées ou ne peuvent être vaccinées pour des motifs médicaux.

4. Décisions non pertinentes. Lorsqu'une décision de refus de la vaccination s'appuie sur des motifs ou des principes qui n'ont pas de rapport avec le maintien de la santé, on peut la considérer comme échappant à la portée des mandats typiques en santé publique, sur la base des principes démocratiques reconnus et des droits des personnes et des groupes. Aux fins du présent ouvrage, on notera particulièrement que les personnes et les groupes peuvent s'opposer à la vaccination en invoquant des valeurs (par exemple leurs croyances d'ordre spirituel ou religieux) qu'ils placent au-dessus de considérations de santé. II faudra peut-être trouver d'autres moyens d'engager un dialogue avec ces groupes.

On ne peut pas douter que tous les motifs de réticence ou d'opposition à la vaccination seront sources de problèmes dans la perspective de santé publique. Cependant, la recherche d'une solution aux enjeux de logistique et d'éthique inhérents aux trois premières catégories relève toujours des responsables de santé publique. C'est le dernier type - sur lequel porte principalement cet ouvrage - qui pose le plus de problème dans la perspective de santé publique, car y trouver une réponse échappe à la portée des mandats typiques dans ce domaine. 


\section{La perspective de la communication du risque}

De nombreux comportements, dans le domaine de la santé, s'expliquent par un paradigme dominant fondé sur les modèles cognitifs dans la prise de décisions ( $\mathrm{v}$. Brunk, chapitre 3 du présent ouvrage). Dans ces approches, les individus sont des décideurs " rationnels » qui tiennent compte des risques et des bienfaits d'événements ou de gestes et choisit la décision qui optimise les avantages (réels ou potentiels) pour lui-même. Dans la foulée, de nombreuses études se sont concentrées sur l'utilisation de ces modèles pour comprendre le recul des taux de vaccination. Selon les modèles cognitifs, dans le contexte d'une décision de vaccination, les facteurs dont on suppose que l'individu tiendrait compte sont notamment la probabilité et la gravité de la maladie, l'efficacité du vaccin et la probabilité qu'il entraîne des réactions indésirables. Dans cette perspective, les problèmes surgissent quand l'individu fonde sa décision sur une surestimation de la probabilité de réactions indésirables, une sousestimation de la gravité de la maladie, etc. Une décision prise sur la foi de telles perceptions ou sentiments est considérée comme «irrationnelle » par le personnel de santé publique, ainsi que par ceux qui ont choisi de promouvoir en priorité ces modèles.

Dans l'application de ces modèles, on suppose qu'une « information correcte sur les risques » provient d'analyses d'experts et tient compte des résultats d'études épidémiologiques et autres de large portée. Lorsqu'il est question de programmes nationaux de vaccination, ces analyses, dans virtuellement la totalité des cas, montrent le caractère rationnel de la décision favorisant la vaccination (sinon, différents vaccins ne seraient sans doute pas offerts dans le cadre de programmes de grande envergure). Selon cette perspective, on arrive à la conclusion générale que les personnes qui ne participent pas aux programmes de vaccination sont mal informées, aboutissent à des conclusions incorrectes et finissent par agir de manière irrationnelle. Suivant ce raisonnement, la réponse politique logique consiste à informer la population, de manière objective, sur les risques des maladies et les risques et bienfaits des vaccins, puisque de cette façon les individus arriveront à la conclusion rationnelle, soit qu'il est dans leur intérêt de se faire vacciner. Non seulement une meilleure information du public est-elle la réponse logique dans cette perspective, mais c'est aussi la réponse éthique, car elle permettra aux individus de prendre de meilleures décisions, qui iront au bout du compte dans leur propre intérêt.

Cette approche permet de mieux comprendre comment les gens prennent des décisions, mais il y a d'autres facteurs à considérer. Premièrement, les parents prennent des décisions, en matière de vaccination, non seulement pour eux-mêmes, mais aussi pour leurs enfants. Dans cette approche globale, les principes généraux sont néanmoins utilisés de manière similaire dans les deux cas. Deuxièmement, les approches cognitives pour la prise de décisions tiennent compte du fait que la vaccination n'est pas indiquée pour certaines personnes, en raison de conditions médicales. De telles considérations ont préséance sur les statistiques démographiques lorsqu'il s'agit de déterminer la probabilité de réactions indésirables; c'est pourquoi on accepte que ces personnes prennent une décision rationnelle en refusant la vaccination. 
Les modèles de prise de décisions qui se préoccupent principalement de l'évaluation du risque et des bienfaits par l'individu désirant optimiser les résultats en matière de santé sont à la fois descriptifs et normatifs. On les dit descriptifs car ils présentent un moyen de comprendre les décisions humaines. Ils sont aussi normatifs du fait qu'ils qualifient les décisions comme «bonnes » ou rationnelles ou comme «erronées » ou irrationnelles. Dans notre cas particulier, en l'absence de troubles médicaux inhabituels (par exemple, une allergie grave aux composants d'un vaccin), une décision de refus de la vaccination est perçue comme irrationnelle. Ce point de vue entraîne un corollaire important, à savoir qu'une partie du risque d'événements indésirables est vue comme acceptable et donc supplantée par les avantages de la vaccination.

Cette approche nous permet de mieux comprendre les répercussions potentielles de croyances erronées au sujet de l'incidence et de la gravité de la maladie. On a prétendu, par exemple, qu'à cause d'une couverture vaccinale élevée, le souvenir d'une maladie infectieuse s'est effacé de la conscience collective et que, simultanément, l'attention a été dirigée vers les risques associés aux vaccins, souvent rapportés faussement (Omer et coll., 2009). On peut donc dire qu'une meilleure couverture vaccinale de la population peut, paradoxalement, entraîner une surestimation du risque du vaccin et une sous-estimation du risque de la maladie. Nous rappelons encore que le plus puissant impératif éthique que puissent produire les différentes approches de compréhension des décisions de vaccination qui sont basées sur la détermination du risque réside dans le fait que l'intérêt des individus est optimisé lorsque ceux-ci reçoivent l'information la plus précise possible sur l'estimation du risque de la vaccination et de la maladie.

En revanche, les approches qui ne font guère plus qu'étudier les processus cognitifs individuels de détermination des risques sont limitées d'au moins quatre manières. Premièrement, elles comportent, comme hypothèse implicite, que les individus agissent afin d'optimiser les résultats à l'échelle de la santé (pour eux-mêmes ou pour leurs enfants). Comme nous l'expliquerons ci-dessous, cette hypothèse n'est pas toujours exacte. Deuxièmement, même si on suppose que les individus prennent des décisions, relativement à la vaccination, qui sont conformes aux objectifs d'optimisation pour la santé, il se peut que les obstacles à l'acceptation ne soient pas de nature cognitive (une estimation erronée des risques, par exemple), mais plutôt d'ordre émotionnel, financier ou pratique (voir le chapitre 6 du présent ouvrage sous la plume de Guay, Dubé et Laberge). En troisième lieu, il est possible que les individus fassent des jugements de valeur au sujet de l'acceptabilité de certains risques, indépendamment de leur probabilité ( $v$. Brunk, au chapitre 3 du présent ouvrage). Quatrièmement (et cette limitation correspond de plus près à notre contexte), ces modèles ignorent et ne reconnaissent pas les aspects culturels et religieux complexes des décisions en matière de vaccination.

Pour ces différentes raisons, d'importantes dimensions de ces décisions passent inaperçues lorsqu'on utilise un modèle cognitif pour les analyser. C'est pourtant un aspect critique de l'évaluation des défis à relever sur le plan éthique quand il est question de réticence à la vaccination. Comme il arrive souvent que l'on n'étudie que les facteurs cognitifs, la portée de l'analyse des répercussions sur le plan éthique est également restreinte. Comme nous l'avons précisé ci-dessus, il s'ensuit que la réponse à l'hésitation, sur ce même plan, consiste simplement à donner une information plus complète et plus précise sur les risques. Examinons 
maintenant deux autres perspectives permettant de comprendre la réticence et l'opposition à la vaccination et permettant d'envisager un éventail plus vaste de facteurs.

\section{La perspective de la psychologie de la santé}

Les approches centrées sur des valeurs heuristiques et des distorsions de nature cognitive représentent un important élargissement des modèles de prise de décision rationnels " classiques ». Dans le chapitre 3 du présent ouvrage, Brunk en fait, dans le contexte des décisions de vaccination, une description et une critique excellentes auxquelles nous n'ajouterons rien ici. Outre ces approches, de nombreux écrits, provenant principalement du domaine de la psychologie de la santé, considèrent que les décisions relatives aux comportements en matière de santé relèvent de facteurs autres que ceux qui découlent du traitement cognitif des risques. Les psychologues de la santé utilisent souvent le modèle de prise de décision individuel décrit précédemment lors de l'étude des décisions de vaccination, mais bon nombre d'entre eux mentionnent aussi des facteurs autres que cognitifs, comme l'inquiétude, le regret, la peur et l'absence de gestion de la douleur. Chapman et Coups (2006), par exemple, ont observé que des réponses émotionnelles à de potentiels événements négatifs (liés au vaccin gratuit contre la grippe, dans leur cas) ont plus d'influence sur une décision que le calcul mathématique des risques. En ce qui concerne la vaccination des enfants, une étude ethnographique relatant des témoignages de mères britanniques au sujet du vaccin RRO (rougeole, rubéole, oreillons) a retrouvé chez plusieurs d'entre elles des « caractéristiques particulières de manies et des phobies » qui auraient influé sur leurs perceptions négatives à l'égard des interventions médicales (Poltorak et coll., 2005). On peut donc conclure qu'une décision de refus de la vaccination, chez un individu, est le résultat de son engagement subjectif dans sa réalité expérientielle (ou celle de son enfant, dans le cas d'une immunisation pédiatrique), plutôt que d'une évaluation non émotive des risques et avantages $^{3}$. Dans les paragraphes qui suivent, nous examinons brièvement quelques-uns de ces éléments subjectifs et passons en revue les considérations éthiques qui accompagnent une approche de la réticence à la vaccination selon ce que nous appelons la perspective de la psychologie de la santé. Nous nous pencherons en particulier sur le rôle que jouent la douleur et la peur inhérentes à la procédure, sur la croyance que certains vaccins entraînent des effets indésirables graves et sur les systèmes de croyances relatifs à la santé alternatifs.

À l'heure actuelle, le calendrier de vaccination recommandé par le Comité consultatif national de l'immunisation prévoit, pour les enfants âgés de moins de six ans, environ quinze vaccinations différentes (y compris le vaccin antigrippal). Les parents doivent donc à de nombreuses reprises donner leur consentement pour que leurs enfants soient vaccinés. Dans un récent sondage mené auprès de 1700 parents canadiens, 89 p. cent ont déclaré que leurs enfants avaient reçu tous les vaccins recommandés, même si 31 p. cent estimaient qu'ils en recevaient trop (Les Associés de recherche EKOS inc., 2011). Pour bien comprendre les divers points de vue et enjeux éthiques (notamment le consentement et l'autorisation) de la vaccination des enfants, il est essentiel de considérer les perspectives et les expériences des parents autant que celles des enfants. La souffrance et la peur sont deux éléments

3. Un parent a cependant déclaré : «Nous, comme parents, ne pourrions pas nous le pardonner si un de nos enfants tombait gravement malade alors que nous aurions pu l'éviter » (Guay et coll., 2015). 
fondamentaux de l'expérience physique et émotionnelle de la vaccination et l'une et l'autre sont subjectives. La détresse procédurale (souffrance et peur) infligée à l'enfant peut et doit exercer une influence sur l'expérience parentale lors de la vaccination et sur les taux d'adhésion. Dans une récente étude sur le rôle de l'expérience de la vaccination dans l'acceptation à l'échelle pédiatrique, par exemple, 16 p. cent des parents ont considéré cette expérience comme négative (Stockwell et coll., 2011). L'origine la plus courante (environ 40 p. cent) de cette situation réside dans la réaction émotionnelle et physique de l'enfant; de tous ces cas, plus de 70 p. cent se rapportaient à une détresse émotionnelle (Stockwell et coll., 2011). On a pu ensuite établir un lien entre les expériences négatives et la non-adhésion : les nourrissons dont les parents avaient éprouvé une expérience négative à la vaccination affichaient un risque deux fois plus élevé de ne pas être adéquatement vacciné (correspondant à un retard d'un mois par rapport au calendrier). Une autre étude, réalisée auprès de mères autochtones canadiennes, a donné un résultat similaire : 100 p. cent de l'échantillon a trouvé difficile d'assister à la détresse de leur enfant pendant la vaccination; de plus, certaines participantes ont indiqué que leur inquiétude, pour ce nourrisson, provenait de la peur des aiguilles chez leurs enfants plus âgés (Tarrant et Gregory, 2003; v. Hobson-West, 2003).

Il est important de savoir qui signale une expérience négative et à quelle fréquence cela se produit. Les enfants, quel que soit leur âge, déclarent avoir très peur des injections et de la douleur qu'elles causent (Hart et Bossert, 1994; Taddio et coll., 2012). Certains professionnels de la santé reconnaissent également cette peur et cette douleur chez l'enfant vacciné (Brady, Avner et Khine, 2011) : les résultats d'une étude menée auprès de groupes de discussion formés d'infirmières en santé publique canadiennes ont fait ressortir que celles-ci connaissaient bien le problème de la crainte et de la douleur causées par les injections et son importance auprès des enfants (Kikuta et coll., 2011). D'un autre côté, la permissionde l'enfant ne semble pas causer de problème pour les prestataires de soins de santé, sous réserve du consentement des parents. Les mères déclarent souvent qu'assister à l'immunisation de leur enfant est une source de détresse (Parvez et coll., 2010; Poltorak et coll., 2005; Tarrant et Gregory, 2003) et il leur arrive de faire une analyse du coût de la vaccination, soit la douleur et la crainte qu'engendre celle-ci (et qu'elles supposent de courte durée), et des avantages qu'elle apporte à long terme (Parvez et coll., 2010). Malheureusement, des interventions psychologiques (une distraction, p. ex.), physiques (meilleur positionnement de l'enfant, etc.) et pharmacologiques (anesthésique topique et autres) sont rarement utilisées (Taddio et coll., 2009, 2010). Les parents, les professionnels de la santé et les enfants reconnaissent généralement la présence d'une douleur, mais celle-ci est fréquemment partiellement gérée ou totalement négligée.

Au moment de prendre une décision de vaccination, porte-t-on attention à la douleur et à la crainte non gérées qu'entraîne la procédure? Dans un cycle négatif, la crainte peut avoir une incidence sur la perception de la douleur (Bird et McMurtry, 2012). La crainte et la détresse non gérées qui accompagnent une procédure médicale douloureuse (comme la vaccination) ont des conséquences à court et à long terme; elles prolongent la durée de la procédure, accroissent la détresse aux procédures suivantes et incitent à éviter les soins de santé (Taddio et coll., 2009). Dans une récente étude canadienne, on a découvert que la peur des aiguilles est courante autant chez les enfants (63 p. cent des jeunes de 5 à 17 ans) que chez les adultes ( 24 p. cent) et qu'elle est la principale raison mentionnée par les parents (7 p. cent) et les enfants (8 p. cent) pour appuyer une non-adhésion à la vaccination (Taddio et coll., 
2012) ${ }^{4}$. Malheureusement, la douleur et la crainte sont généralement peu mentionnées dans les discussions entourant la vaccination. Citons en exemple le sondage auprès de parents canadiens mentionné précédemment (Les Associés de recherche EKOS inc., 2011); on y voit que 10 p. cent des parents ont choisi de refuser la vaccination pour les raisons suivantes : 28 p. cent la trouvaient non nécessaire, 17 p. cent doutaient de son innocuité, 16 p. cent la croyaient inefficace, 12 p. cent estimaient les effets secondaires trop nombreux et 3 p. cent, ayant déjà manqué une immunisation, ont donné une réponse plus tard classée dans la catégorie : « II y a trop d'injections et cela traumatise mon enfant ». On a cependant éliminé la deuxième partie de la réponse ( et cela traumatise mon enfant») lors de la publication des résultats et nulle part ailleurs dans le document les administrateurs du sondage n'ont mentionné le mot «douleur ». La non-reconnaissance de la douleur et de la crainte comme facteurs des décisions de vaccination et la rareté des efforts d'intervention pour les apaiser incitent à se demander si le peu d'attention apparent, de la part des professionnels de la santé, ne va pas affecter la confiance que les adultes leur portent et leur désir de consentir à la vaccination au nom de leur enfant. On peut certes affirmer que les expériences médicales passées et les relations antérieures avec des professionnels de la santé risquent d'avoir une influence sur la confiance d'un individu en la vaccination (se reporter par exemple à Poltorak et coll., 2005). Stockwell et ses collègues (2011) ont démontré que le deuxième motif le plus important signalé par les mères pour désigner comme négative une expérience de vaccination de leur enfant réside dans l'attitude du personnel des soins de santé. II est donc possible que les perceptions quant à cette attitude, en ce qui concerne l'effet immédiat de la vaccination, puissent avoir un rôle à jouer dans la réflexion des parents, au sujet de leur consentement. Dans des cas extrêmes, les parents qui perçoivent une insensibilité du personnel clinique pourraient perdre confiance dans l'innocuité et l'efficacité générales des vaccins, en plus d'avoir l'impression d'être mal informés au moment de donner leur consentement; en outre, l'enfant qui montre clairement sa détresse à la vue d'une aiguille peut exprimer ainsi un refus d'être vacciné très évident. Stockwell et ses collègues (2011) recommandaient donc que le personnel clinique offre un soutien aux parents et assure une meilleure gestion de la peur et de la douleur pour accroître l'acceptation des vaccins chez les enfants.

Dans la compréhension des perspectives associées à la vaccination, un autre domaine mérite une étude plus approfondie : il s'agit des croyances en matière de santé et des autres systèmes de croyance qui ont une incidence sur les décisions, que ces croyances soient caractérisées comme cognitives ou émotionnelles. Les croyances qui font de la vaccination la cause d'événements ou de réactions indésirables devraient probablement faire l'objet d'une attention particulière. La croyance voulant que le vaccin RRO, par exemple, soit à l'origine de l'autisme a entraîné une diminution significative de l'acceptation de ce vaccin et un retard des parents à la vaccination de leur enfant. La prétention voulant que le vaccin RRO cause l'autisme a été fortement médiatisée et cela a pu, en conséquence, la doter d'une crédibilité ou d'une légitimité chez certains individus (Woo et coll., 2004). L'Institute of Medicine a passé l'étude en revue et n'a découvert aucune donnée probante pouvant établir un lien entre le vaccin et l'autisme (Institute of Medicine, 2001, 2004); à cela s'ajoute le fait que, comme Bramadat (voir le chapitre 1), Brunk (chapitre 3), Boucher (chapitre 11) et Bramadat, Bettinger et Guay (chapitre 14) l'expliquent dans le présent ouvrage, l'étude et le chercheur (Wakefield)

4. En fait, cette crainte agit souvent par «procuration »; comme déclarait un parent : "Ma crainte pour mon enfant est plus grande que la sienne. Elle provient du fait qu'il a mal au moment de l'injection » (Guay et coll., 2015). 
qui avaient initialement donné à ce lien une certaine légitimité, ont tous deux été discrédités. La croyance voulant que le vaccin RRO cause l'autisme n'est malgré tout pas disparue et joue encore un rôle évident dans les décisions de vaccination'; certains en font une espèce d'acte de foi n'ayant pas de relation avec les données réelles.

Dans certains modèles biomédicaux maintenant dépassés, la santé était implicitement vue comme représentant l'absence de maladies. De nos jours, des modèles plus élargis gagnent en popularité. L'approche « biopsychosociale » est réputée convenir mieux à la compréhension et au traitement des maladies chroniques ou de celles qui sont fortement présentes dans la société d'aujourd'hui (cancer, maladie cardiaque, diabète, etc.). La médecine complémentaire et alternative et l'approche holistique de la santé gagnent aussi en popularité. Comme le suggèrent plus loin Guay, Dubé et Laberge (chapitre 6) et Dubé, Sauvageau et Gagnon (chapitre 7), de nombreux patients qui s'intéressent à l'homéopathie, à la chiropratique et à la naturopathie et de nombreux cliniciens qui les pratiquent ont une attitude critique à l'égard de la vaccination (v. Ernst, 2001). Dans leurs cas, le nœud du problème ne réside pas dans le fait que les personnes comprennent mal les risques inhérents à leur décision; c'est plutôt qu'au rythme de la popularité et de l'acceptation croissante de ces approches, on assistera à une profonde remise en question des discours sur la santé accessibles au public (HobsonWest, 2003).

Somme toute, contrairement à bien des approches de communication des risques, les modèles de décision individuels en matière de vaccination peuvent être établis de manière à tenir compte de facteurs allant au-delà du traitement de l'information cognitive et prenant en considération des réponses émotionnelles et physiologiques interdépendantes (crainte et douleur), des réactions de parents à la crainte et à la douleur manifestées par leur enfant, des croyances particulières à l'effet que les vaccins causent des problèmes (par exemple le vaccin RRO et l'autisme) et des systèmes de croyance élargis en matière de santé (par exemple la médecine complémentaire et alternative). Heureusement, pour les partisans de la vaccination, l'accroissement de la gamme des facteurs reconnus comme influençant les décisions multiplie le nombre de cibles d'intervention possible. En ce qui concerne les personnes ayant peur des aiguilles (Taddio et coll., 2012), par exemple, des interventions efficaces pourraient être conçues pour promouvoir les pratiques d'immunisation qui gèrent mieux la douleur provoquée par les injections.

Quelles sont les dimensions éthiques de décisions de vaccination dans la perspective de ce que nous appelons la perspective de la psychologie de la santé? Quelle est, en particulier, la signification éthique de tentatives d'accroître les taux de vaccination en composant mieux avec la crainte et la douleur que celle-ci entraîne ou d'efforts visant à modifier les croyances individuelles? On dira peut-être que, rien qu'en s'attaquant au problème de la crainte et de la souffrance, on exerce un certain degré de paternalisme; il est rare cependant que l'on soulève des questions éthiques pour ce genre d'intervention. Autrement dit, compte tenu de l'objectif élargi de promotion de la santé individuelle et publique, il semble raisonnable de réduire la douleur causée par la procédure et d'aider les personnes à surmonter certains obstacles comme la peur des aiguilles, même si cette assistance n'est pas demandée. À l'opposé, on peut associer de plus grands enjeux éthiques à la volonté de modifier les croyances populaires

5. Une des participantes à l'étude Guay et coll. (2015) a noté qu'elle est préoccupée par le « vaccin RRO, car c'est un vaccin vivant et qu'il comporte des risques neurologiques; je manque aussi d'information sur le lien avec l'autisme». 
au sujet du corps humain ou de l'univers dans le but de hausser les taux de vaccination. Puisque la plupart des pays développés sont en faveur de la liberté de religion, toute tentative de modifier ces croyances dans le cadre d'une campagne de santé publique devrait faire l'objet de délibérations exhaustives entre les politiciens, les professionnels de de la santé et les fidèles de groupes religieux. En fait, ce devrait être le cas de la quasi-totalité des critiques de croyances imbriquées dans les médecines complémentaires et alternatives, car ces pratiques découlent, la plupart du temps, non pas d'allégations vérifiables de manière empirique, mais de systèmes de croyance philosophiques plus étendus qui s'intéressent à la relation entre l'homme et un plus ample ordre cosmique.

La perspective de la psychologie de la santé, se distinguant ainsi des perspectives de la communication du risque et de santé publique, comporte un moyen de comprendre une large gamme de facteurs ayant une incidence sur les décisions de vaccination. Elle offre donc également un ensemble plus étendu d'interventions possibles, en cas de réticence ou de résistance à la vaccination. Ces interventions ne sont cependant pas toutes acceptables d'un point de vue éthique ou politique, du moins dans l'environnement sociopolitique actuel. Dans nos sociétés multiculturelles et pluralistes, nous nous sommes engagés à respecter une vaste gamme de croyances et de cosmologies culturelles et religieuses. Sur le plan éthique, il peut être difficile de plaider en faveur d'une modification des croyances culturelles et religieuses d'une population à des fins de santé publique, même si ces croyances vont de pair avec une décision de refus de la vaccination.

\section{La perspective socioculturelle}

Le risque est le concept unissant de nombreuses approches de compréhension de la résistance à la vaccination. Hobson-West (2003) plaide de manière convaincante, à ce sujet, qu'il ne faut pas faire du risque le principal outil conceptuel de compréhension et encore moins de recherche de solutions, en matière de résistance à la vaccination. Dans les pages qui précèdent, nous avons déjà indiqué que certains facteurs non liés à la perception du risque - comme la peur des aiguilles ou un engagement en faveur de philosophies alternatives de soins de santé - ont un grand rôle à jouer dans les décisions de vaccination. Nous allons maintenant nous pencher sur les questions qui transcendent la perception individuelle du risque. Bien que nous ayons jusqu'ici ciblé la prise de décision individuelle dans nos efforts de compréhension de la résistance et la réticence à la vaccination, nous pouvons également dire qu'un objectif clé des programmes de vaccination consiste à protéger les populations et peut-être même la société tout entière. Par ailleurs, la résistance à la vaccination prend souvent racine dans des perspectives religieuses, des systèmes de croyance et des pratiques culturelles de groupes et de collectivités et non pas chez les citoyens pris isolément. II est donc essentiel de porter son attention sur les perspectives dont la portée sociale, culturelle et politique est la plus grande. Nous tenterons, dans les paragraphes qui suivent, de démontrer que, pour certains groupes et individus, la vaccination peut acquérir une valeur symbolique transcendant le concept du risque et incitant au refus de l'immunisation. Pour certaines collectivités religieuses, la vaccination est parfois le symbole d'une société de plus en plus acquise à la laïcité et qui se montre hostile aux allégations religieuses ou spirituelles; ce phénomène est observable non seulement au Nigeria, en Afghanistan et au Pakistan (dont Bramadat a traité au chapitre 1), 
mais aussi chez les fidèles de l'Église réformée néerlandaise au Canada. Pour d'autres, la vaccination représente, pour la société, un moyen de conserver le contrôle sur des minorités raciales ou ethniques; d'autres enfin la considèrent comme l'outil d'un gouvernement toutpuissant désireux (sous l'impulsion des grands fabricants de médicaments) d'exercer le contrôle absolu sur ses citoyens ${ }^{6}$.

La résistance aux programmes de vaccination ne se manifeste pas au hasard, ni n'est uniformément répartie dans la population. Elle peut être associée à des groupes ou des segments de la société et même, dans certains cas, à des régions ou à des quartiers. La relation entre le statut social et l'attitude à l'égard de la vaccination compte parmi les facteurs importants à considérer dans l'étude de ces associations. Au Royaume-Uni, par exemple, les autorités de santé publique et l'élite de la société ont accueilli des lois favorisant la vaccination et en ont fait un objet de fierté nationale, mais, chez les travailleurs et les membres de la classe moyenne, nombreux étaient ceux qui se méfiaient des lois adoptées entre 1840 et 1898 et de leurs répercussions sur leur vie quotidienne (voir MacDougall et Monnais, au chapitre 5 du présent ouvrage). De cruelles pratiques discriminatoires étaient chose courante et les autorités visaient fréquemment la classe ouvrière pour assurer l'exécution de ces lois, la voyant comme source de la maladie (Durbach, 2000). Les autorités chargées de la vaccination avaient le droit, en vertu de la législation, de repérer les contrevenants et de mettre à l'amende ou emprisonner les parents qui refusaient de se soumettre; il était notoire que l'on était moins exigeant à l'égard des classes moyenne et supérieure (Durbach, 2000; Wolfe et Sharp, 2002). La classe ouvrière considérait la vaccination obligatoire comme un outil législatif visant implicitement les jeunes enfants de travailleurs et assorti de pénalités inéquitables [Durbach, 2000; Stern et Markel, 2005; se reporter également à Roy (chapitre 4) et MacDougall et Monnais (chapitre 5) pour une étude de la situation contemporaine au Canada], tandis que la classe moyenne y voyait l'imposition de contraintes à l'individualisme et à la liberté.

Une agitation croissante, dans la classe ouvrière et la classe moyenne, a mené à la naissance d'un mouvement anti-vaccination très déterminé, voué à la contestation des lois et à la défense des droits de ses membres et de l'intégrité du corps des enfants. La pression a monté jusqu'à ce que le mouvement atteigne un certain succès avec l'intégration de la clause des « objecteurs de conscience » en 1898; les parents avaient dès lors une possibilité de refus s'ils estimaient les vaccins inefficaces ou dangereux (Wolfe et Sharp, 2002). En Occident, la réponse à la vaccination a été complexe tout au long de l'histoire; rappelons-nous cependant que la contestation du premier mouvement anti-vaccination demeure pertinente encore aujourd'hui, car les croyances et attitudes fondamentales n'ont pas été démenties en près de deux siècles et sont toujours partagées par les opposants actuels [Wolfe et Sharp, 2002; v. Durbach, 2000; Roy (chapitre 4) et MacDougall et Monnais (chapitre 5)].

Outre la lutte des classes et l'équité sociale, la religion est parfois liée à la position des opposants à la vaccination. Comme nous l'avons vu précédemment en étudiant les cas où la promotion de la vaccination par les autorités de santé publique est remise en question par des groupes religieux, on ne signale généralement pas de désaccord précis sur les risques liés aux vaccins ou sur les maladies que ces derniers doivent prévenir. La contestation semble plutôt provenir d'un conflit entre, d'un côté, la vaccination et, de l'autre, les pratiques ou

6. Un parent a dit : "Je ne crois pas que les vaccins soient nécessaires et les études et les recommandations du gouvernement ne m'inspirent pas confiance; j'entretiens des doutes au sujet des fabricants de médicaments, dont l'intérêt financier les incite à vendre leurs vaccins » (Guay et coll., 2015). 
croyances du groupe religieux visé. En Amérique du Nord, divers groupes religieux comme Faith Assembly, Faith Tabernacle, End of Time Ministries, les huttérites et l'église de la Science chrétienne ont adopté des systèmes de croyance qui vont à l'encontre de bien des découvertes médicales, y compris la vaccination, et même en encouragent le rejet. Les fidèles de l'église de la Science chrétienne, par exemple, essaient de régler des problèmes de santé par la prière, convaincus de la bonté de Dieu et de la volonté de celui-ci de garder chacun de nous en santé pour la vie (voir «What Is Christian Science? », 2011). D'autres groupes religieux considèrent aussi la vaccination comme un obstacle à la volonté de Dieu (Kulig et coll., 2002; v. Bramadat, introduction et chapitre 1 du présent ouvrage).

Dans certains cas, la réticence à la vaccination parmi les groupes religieux peut être liée à des croyances qui, sans être vraiment des éléments d'une doctrine officielle, circulent dans des communautés isolées et ont une incidence sur la réponse à la vaccination. Les huttérites, par exemple, professent des croyances au sujet de la volonté de Dieu qui détermine l'état de santé de tous les êtres humains, mais croient aussi que les vaccins ne réussissent pas à protéger l'homme contre les maladies, voire qu'ils peuvent lui nuire (Kulig et coll., 2002). De la même façon, certains ont tenté d'expliquer la faible acceptation de la vaccination pédiatrique chez les juifs orthodoxes en invoquant une crainte exagérée de réactions indésirables perdurant dans le groupe (Loewenthal et Bradley, 1996).

On a aussi noté des taux d'acceptation peu élevé au sein de certaines minorités raciales et ethniques (Dhami et Sheikh, 2008; voir également Guay, Dubé et Laberge, au chapitre 6); ils pourraient être associés à des enjeux d'équité en santé. Fitch et Racine (2004) signalent qu'en matière d'immunisation des enfants, une majorité de parents habitant des quartiers urbains mal desservis et relativement pauvres croyait que la vaccination était importante pour la santé, mais entretenait de fausses idées (relativement aux communications officielles de santé publique) sur la nécessité de suivre le calendrier recommandé, sur les effets que pourraient subir les systèmes immunitaires des enfants et sur l'innocuité de la procédure. On pourrait attribuer de tels préjugés à un manque de sensibilisation et d'information sur les vaccins. Dans un autre exemple, un groupe de discussion organisé par Daniels et ses collègues (2004) a suggéré que les participants issus de minorités raciales et ethniques de San Francisco étaient mal informés des bienfaits et des effets indésirables potentiels des vaccins contre la grippe et les infections à pneumocoques. Dans une autre étude réalisée à Los Angeles et à Honolulu, on a découvert que la vaccination contre la grippe était beaucoup plus rare chez les Afro-américains et les personnes d'origine latino-américaine que chez les personnes de race blanche; selon la réponse donnée le plus souvent par les gens non vaccinés, le vaccin n'était pas nécessaire, puisqu'ils n'étaient pas à risque et utilisaient d'autres moyens de prévention (Chen et coll., 2007). Quoiqu'il faille tenir compte de lacunes d'information et de sensibilisation de certains groupes relativement à l'utilisation et à l'efficacité des vaccins, les principales raisons motivant la faiblesse du taux d'acceptation résident dans un statut socioéconomique relativement faible, la pauvreté et des questions de logistique (voir Guay, Dubé et Laberge, chapitre 6 du présent ouvrage). Comme les calendriers de vaccination exigent souvent de nombreuses administrations sur une assez longue période, il n'est pas étonnant que les couvertures soient plus faibles dans certains groupes minoritaires, puisque les parents ayant plus de ressources (statut socioéconomique plus élevé) seront plus en mesure de respecter les recommandations que les parents moins nantis, qui doivent s'absenter du travail ou se rendre plusieurs fois à la clinique (Omer et coll., 2009). Des taux de vaccination moins élevés ne doivent pas automatiquement être attribués à des facteurs de disposition (par exemple 
des croyances et des attitudes). Ils pourraient être plutôt le résultat des nombreux obstacles complexes qui contribuent à la croissance des disparités en matière de santé (Fiscella et coll., 2000). Par conséquent, dans certaines circonstances, de faibles taux de vaccination se comprennent mieux si on en fait un symptôme d'iniquité, plutôt que de résistance.

L'argument sur lequel nous insistons ici est le suivant: que l'on associe la vaccination à des identités de classe, de race, d'ethnie ou de religion, les décisions prises ne sont pas seulement des décisions individuelles, mais sont fortement liées à l'adhésion au groupe. Bien des groupes mentionnés dans ces pages ont une caractéristique remarquable, à savoir qu'ils se méfient de la société en général et particulièrement de certaines structures d'autorité. Les Afro-américains, par exemple, font l'objet d'une discrimination dans le système de soins de santé des États-Unis et se montrent plus méfiants à l'endroit des organismes gouvernementaux, des chercheurs en médecine et des prestataires de soins de santé (Chen et coll., 2007). Des études réalisées auprès des Premières nations canadiennes ont démontré également que les croyances actuelles allant dans le sens d'un refus de la vaccination peuvent être le résultat de mauvaises expériences passées ayant incité les aînés à cesser d'avoir confiance dans le système de soins de santé (Tarrant et Gregory, 2001). On a retrouvé cette méfiance à l'endroit des professionnels des soins de santé, dans le cas des vaccinations, parmi les mères de famille juives orthodoxes (Loewenthal et Bradley, 1996). Elle ne peut pas être simplement assimilée à des perceptions d'individus pris isolément. Dans bien des cas, on retrouve de longs antécédents, amplement documentés, de discrimination systématique et de pratiques contraires à l'éthique contre ces groupes et qui ressortent clairement de leurs attitudes à l'endroit de la vaccination.

Comme l'explique Brunk au chapitre 3 du présent ouvrage, plus élevé est le degré de méfiance à l'égard des agents de communication et de gestion du risque, moins il est probable que la communication et la persuasion parviendront à convaincre les individus et les groupes des bienfaits de la vaccination. À cet égard, les vaccins ne peuvent pas être vus comme des interventions qui réduisent les risques et sont isolés des autres considérations. II faut plutôt examiner l'acceptation et la réticence à la vaccination en tenant compte du contexte social, culturel et politique où on les constate. Nous avons mentionné précédemment que, selon les circonstances, la réticence à la vaccination d'individus et de groupes peut être le résultat de la résistance à l'évolution vers la laïcité d'une société qui déconsidère les valeurs religieuses, d'une société dominée par la race blanche qui essaie de circonscrire les minorités ou d'un gouvernement tout-puissant qui cherche à contrôler ses citoyens.

En ce qui concerne la confiance, il faut ajouter une considération supplémentaire relative aux systèmes de valeurs dans lesquels sont imbriqués les comportements en matière de vaccination. Les vaccins et les programmes de santé publique dont ils découlent sont au fond basés sur un système de valeurs qui est largement accepté dans la société, mais pas universellement. Nous estimons qu'il faut se garder de contester ces valeurs dominantes. Notre objectif consiste plutôt à souligner qu'on ne peut exclure les valeurs de ces activités et que ces valeurs ne sont peut-être pas partagées dans l'ensemble de la société. Par exemple, comme nous en avons discuté précédemment (voir également Bramadat, dans l'introduction et le premier chapitre du présent ouvrage), certaines croyances religieuses rejettent la vaccination parce qu'elle serait un moyen d'interférer avec la volonté de Dieu. Les politiques liées aux sciences et les défenseurs de santé publique qualifient souvent ces croyances d'« irrationnelles ». Néanmoins, dans de nombreux cas, elles échappent à la portée empirique, car elles ne réfutent pas la validation scientifique de la vaccination (par exemple au sujet de 
son efficacité), ni même ne la contestent, mais elles entraînent les croyants à s'y opposer. Pour mieux les comprendre, il faut donc les voir comme étant le fondement ou le résultat d'un système de valeurs qui s'éloigne du système prévalent sous-tendant les activités de santé publique.

Quelles sont donc les considérations éthiques se rapportant à la réticence à la vaccination, dans la perspective socioculturelle? Quand la vaccination est obligatoire, nombreux sont les gouvernements qui permettent des exemptions, pour une foule de raisons. II n'y aura aucun problème si les exemptions sont rares et bien réparties au sein de la population dans son ensemble. Cependant, comme nous l'avons indiqué précédemment, les représentants de santé publique font face à un dilemme quand le taux de vaccination est très faible et que des personnes vulnérables sont susceptibles de contracter une maladie contagieuse. Les autorités de santé publique se font un devoir de promouvoir la vaccination et prennent des mesures pour en optimiser l'acceptation. Toutefois, lorsque la résistance est fondée sur des systèmes de croyance différents ou rattachée à la race ou à la classe sociale, on peut, dans une perspective politique, imaginer le scénario d'un groupe (plus grand et plus avantagé) désirant imposer sa volonté et ses croyances à un autre (plus petit et moins avantagé). Le problème se présente donc ainsi : « nous » (la majorité) savons ce qui est le mieux pour « eux » (la minorité) et décidons de le lui imposer.

La liberté de religion, considérée comme un droit fondamental garanti dans les démocraties libérales, à l'instar des droits parentaux, n'est cependant pas absolue : les tribunaux ont le pouvoir (et le devoir) de limiter ces droits pour assurer la sécurité publique et protéger la santé des citoyens (Barnett, 2011). La Cour suprême des États-Unis, d'ailleurs, a jugé que l'on n'enfreint pas la liberté de religion garantie par la Constitution en rendant la vaccination obligatoire pour les enfants (Asser et Swan, 1998); la jurisprudence canadienne comprend des affaires dans lesquelles on a établi que l'évaluation du gouvernement, faite au mieux de l'intérêt de l'enfant, avait préséance sur les autres?. Field et Caplan (2008) font aussi remarquer qu'il est dans la nature du gouvernement d'exercer un pouvoir coercitif. Dans bien des cas, les interventions de santé publique ne sont pas, à première vue, coercitives. Par contre, il faut bien voir un certain degré de coercition dans des efforts même moins directs de modification des comportements, tels qu'une restriction de l'accès à l'école, à l'emploi ou aux services de garde d'enfants.

Nous ne recommandons pas d'accorder à tout prix le respect de l'autonomie des individus et des groupes. Au contraire, il est reconnu qu'il y a un conflit entre les droits individuels et les mesures prises dans le cadre de programmes de santé publique et qu'il ne se réglera pas facilement (Siegal, Siegal et Bonnie, 2009). Si l'on adopte une perspective tenant compte de réalités plus larges en matière sociale, culturelle, religieuse et politique, cependant, il est essentiel de reconnaître expressément les valeurs sur lesquelles sont fondés les systèmes de santé publique. Grâce à cette franchise et à cette transparence, on pourra en arriver à un dialogue plus ouvert avec les collectivités dont les systèmes de valeurs différents incitent à la réticence face à la vaccination. Les principes à suivre résident au fond dans l'établissement de relations de confiance entre les travailleurs de la santé et les patients, entre les groupes et au sein de structures sociales élargies. II faudra, une fois les valeurs différentes reconnues, accepter que la résistance à l'égard des pratiques de santé publique puisse être fondée sur une

7. Voir A.C. c. Manitoba (Directeur des services à l'enfant et à la famille) [2009] 2 RSC 181, 2009 CSC 30. https:// scc-csc.lexum.com/scc-csc/scc-csc/fr/item/7795/index.do 
méfiance plus générale à l'endroit du gouvernement et sur un passé et un présent marqués par l'injustice sociale, la discrimination raciale et les inégalités sociales et économiques. Selon les arguments de Wynne (2006), le développement de la confiance doit reposer principalement sur des efforts d'amélioration de la fiabilité des structures d'autorité, et non pas sur des tentatives de la créer au sein de populations ciblées. Selon la perspective politique et socioculturelle, les critères de l'éthique, dans la réaction à la réticence face à la vaccination, exigent que l'on institue un dialogue franc et ouvert et que l'on veille au respect des individus et des groupes qui affichent des valeurs et des expériences sociales conflictuelles. La perspective dominante de la santé a pour caractéristiques principales d'être fondée uniquement sur la science et non sur les valeurs et de tenir pour acquis que le gouvernement est bienveillant. Si nous souhaitons encourager tous les citoyens à se prononcer en faveur de pratiques de santé publique qui avantagent autant les individus que la société en général, ces hypothèses doivent être mises de côté ou franchement remises en question.

\section{Conclusion}

Nousavonsvoulu, dans ce chapitre, examiner les répercussionssur le plan éthique d'une gamme de réactions différentes à la réticence face à la vaccination. Nous avons notamment analysé les aspects éthiques de ce phénomène sur la base de quatre perspectives distinctes (santé publique, communication du risque, psychologie de la santé et perspective socioculturelle) actuellement utilisées dans la controverse entourant la vaccination. Nous avons émis l'opinion que les approches dominantes, en ce qui concerne la compréhension des décisions de vaccination, s'inspirent d'hypothèses cognitivistes et individualistes et ne réussissent pas à saisir toute la portée du phénomène de la réticence et de la résistance à la vaccination. II faudra, pour mieux comprendre d'importants éléments du problème, acquérir des perspectives plus larges. Nous n'affirmons pas que ces perspectives soient les seules pertinentes, ni qu'elles fassent état de tous les dilemmes éthiques que pourrait susciter l'administration des vaccins dans une société pluraliste. Nous croyons cependant que notre analyse met en lumière des enjeux qui méritent plus ample considération. Premièrement, les notions normatives varient selon la position de l'observateur et le cadre de travail théorique adopté. En pratique, cela signifie qu'une réponse politique officielle à la réticence face à la vaccination, pour être vue comme « éthique » par un auditoire élargi, ne doit pas se limiter à considérer le problème d'un seul point de vue (soit habituellement la perspective cognitive de communication du risque). En deuxième lieu, quoique certaines perspectives semblent plus approfondies ou exhaustives que d'autres, nous estimons que celles de santé publique, de la communication du risque, de la psychologie de la santé et de la culture sociale permettent de faire ressortir un plus grand nombre de considérations éthiques dans la réponse à la réticence face à la vaccination. Troisièmement, nous croyons qu'il y aura beaucoup à gagner si les responsables de santé publique utilisent une vaste gamme de perspectives afin de mieux comprendre le problème que représentent l'opposition et la réticence à la vaccination. La perspective de la culture sociale peut être plus ardue à adopter, mais elle aura sans doute l'avantage de proposer des points de vue variés à prendre en considération dans la formulation des interventions et des réponses politiques. C'est probablement la perspective qui comportera une des approches les plus utiles quand il s'agira de répondre à la réticence à la vaccination qui trouve son origine dans les convictions religieuses ou les cosmologies. 
Nous aimerions, en conclusion, formuler quelques réflexions sur la pratique. Notre analyse révèle certains aspects incongrus dans de nombreuses stratégies de promotion de la vaccination par les responsables de santé publique. Nous avons entre autres noté que ces derniers la conçoivent comme un avantage collectif sur le plan de la santé. Toutefois (et, en partie, selon le vaccin en cause), la documentation de promotion semble souvent s'inspirer de cadres de travail individualistes pour la communication du risque et les modèles rationnels de prise de décision. Comme la santé publique vise des objectifs de nature générale, comme l'immunité collective, et que bien des facteurs caractérisant la réticence à la vaccination sont du même ordre, il est possible que les stratégies de promotion visant des groupes et des identités de groupe risquent d'être plus efficaces que celles qui ciblent des risques et des bienfaits pour l'individu. Il est également logique, compte tenu des considérations éthiques, que les individus ayant décidé de refuser la vaccination ne prennent pas cette décision pour eux-mêmes seulement, car ils augmentent ainsi le risque auquel leur collectivité est soumise. La réticence à la vaccination qu'expriment certains individus peut être associée à une identité de groupe donnée (identité religieuse, ethnique ou de croyances holistiques, etc.). Ainsi donc, l'individu dont l'identité religieuse l'incite à résister à la vaccination peut aussi l'accepter parce qu'il est aussi un parent, un citoyen ou un voisin. Autrement dit, l'affinité de l'individu avec une croyance, une pratique ou un groupe ayant une identité discursive ne révèle qu'un aspect de son identité et n'est donc pas le seul facteur déterminant de ses décisions de vaccination. Les stratégies de santé publique qui sont fondées sur une compréhension des multiples et convergentes couches de l'identité pourraient fort bien être les plus efficaces, surtout si elles s'accompagnent de messages qui développent les relations de confiance et l'inclusion entre groupes et entre individus. Par ailleurs, si on a confiance en la vaccination parce qu'elle est recommandée et administrée par une personne «qui me ressemble » (particulièrement un membre du même groupe religieux, ethnique, racial ou culturel) plutôt que par " eux ", il pourrait être possible d'accroître le taux de vaccination tout en favorisant l'autonomie individuelle et de groupe. De la même façon, si on fait la promotion de la vaccination non seulement dans " mon propre intérêt », mais aussi dans l'intérêt de " ma collectivité et de ceux que j'aime », on pourrait peut-être commencer à résoudre la dichotomie entre l'intérêt personnel et le bien-être collectif. 


\section{BIBLIOGRAPHIE}

Anikeeva, O., A. Braunack-Mayer, and W. Rogers. 2009. "Requiring Influenza Vaccination for Health Care Workers." American Journal of Public Health 99 (1): 24-9. http://dx.doi.org/10.2105/AJPH.2008.136440.

Asser, S.M., and R. Swan. 1998. "Child Fatalities from Religion-Motivated Medical Neglect." Pediatrics 101 (4): 625-9. http://dx.doi.org/10.1542/peds.101.4.625.

Barnett, L. 2011. Freedom of Religion and Religious Symbols in the Public Sphere. Ottawa: Library of Parliament, Publication no. 2011-60-E. July 25. Accessed 15 May 2014, http://www.parl.gc.ca/Content/LoP/ ResearchPublications/2011-60-e.htm\#a5.

Bird, L., and C.M. McMurtry. 2012. "Fear in Pediatric Acute Pain: Role and Measurement." Pain Management 2 (6): 527-9. http://dx.doi.org/10.2217/pmt.12.56.

Brady, K.A., J.R. Avner, and H. Khine. 2011. "Perception and Attitude of Providers toward Pain and Anxiety Associated with Pediatric Vaccine Injection." Clinical Pediatrics 50 (2): 140-3. http://dx.doi. org/10.1177/0009922810384721.

Centers for Disease Control and Prevention (CDC). 2011. "Immunization of Health-Care Personnel: Recommendations of the Advisory Committee on Immunization Practices." Morbidity and Mortality Weekly Report (MMWR) 60 (RR07): 1-45. http://www.cdc.gov/mmwr/preview/mmwrhtml/rr6007a1.htm.

Chapman, G.B., and E.J. Coups. 2006. "Emotions and Preventive Health Behavior: Worry, Regret, and Influenza Vaccination." Health Psychology 25 (1): 82-90. http://dx.doi.org/10.1037/0278-6133.25.1.82.

Chen, J.Y., S.A. Fox, C.H. Cantrell, S.E. Stockdale, and M. Kagawa-Singer. 2007. "Health Disparities and Prevention: Racial/Ethnic Barriers to Flu Vaccinations." Journal of Community Health 32 (1): 5-20. http:// dx.doi.org/10.1007/s10900-006-9031-7.

Daniels, N.A., T. Juarbe, M. Rangel-Lugo, G. Moreno-John, and E.J. Pérez-Stable. 2004. "Focus Group interviews on Racial and Ethnic Attitudes Regarding Adult Vaccinations." Journal of the National Medical Association 96 (11): 1455-61.

Dhami, S., and A. Sheikh. 2008. "Health Promotion: Reaching Ethnic Minorities." Practice Nurse 36 (8): 21.

Durbach, N. 2000. "They Might As Well Brand Us: Working-Class Resistance to Compulsory Vaccination in Victorian England." Social History of Medicine 13 (1): 45-63. http://dx.doi.org/10.1093/shm/13.1.45.

Ekos Research Associates, Inc. 2011. Survey of Parents on Key Issues Related to Immunization. Final Report Submitted to the Public Health Agency of Canada. September. Accessed 15 May 2014, http:// resources.cpha.ca/immunize.ca/data/1792e.pdf.

Ernst, E. 2001. "Rise in Popularity of Complementary and Alternative Medicine: Reasons and Consequences for Vaccination." Vaccine 20 (Suppl 1): S90-3. http://dx.doi.org/10.1016/S0264-410X(01)00290-0.

Field, R.I., and A.L. Caplan. 2008. "A Proposed Ethical Framework for Vaccine Mandates: Competing Values and the Case of HPV." Kennedy Institute of Ethics Journal 18 (2): 111-24. http://dx.doi.org/10.1353/ ken.0.0011.

Fiscella, K., P. Franks, R.M. Gold, and M.C. Clancy. 2000. "Inequality in Quality: Addressing Socioeconomic, Racial, and Ethnic Disparities in Health Care." Journal of the American Medical Association 283 (19): 2579-84. http://dx.doi.org/10.1001/jama.283.19.2579.

Fitch, P., and A. Racine. 2004. "Parental Beliefs about Vaccination Among an Ethnically Diverse Inner-City Population." Journal of the National Medical Association 96 (8): 1047-50. 
Guay M., E. Cadieux, J. Désilets, P. Clément, C. Vanier, S. Briand, J. Lemaire, C. Michaud, E. Dubé, C. Boulet, N. Boulianne, and M. Landry. 2015. Quel est le meilleur mode d'organisation de la vaccination des enfants de 0-5 ans au Québec? Enquête auprès des parents. Quebec: Institut national de santé publique du Québec. En rédaction.

Hart, D., and E. Bossert. 1994. "Self-Reported Fears of Hospitalized School Age Children." Journal of Pediatric Nursing 9: 83-90.

Hobson-West, P. 2003. "Understanding Vaccination Resistance: Moving Beyond Risk." Health Risk \& Society 5 (3): 273-83. http://dx.doi.org/10.1080/13698570310001606978.

Institute of Medicine. 2001. Immunization Safety Review: Measles-Mumps-Rubella Vaccine and Autism. Washington, DC: National Academy Press. Accessed 12 May 2014, https://www.nap.edu/catalog/10101/ immunization-safety-review-measles-mumps-rubella-vaccine-and-autism.

- 2004. Immunization Safety Review: Vaccines and Autism. Washington, DC: National Academies Press. Accessed 17 May 2014, https://www.nap.edu/catalog/10997/ immunization-safety-review-vaccines-and-autism.

Isaacs, D.D., H.A. Kilham, J.J. Leask, and B.B. Tobin. 2009. "Ethical issues in Immunisation." Vaccine 27 (5): 615-8. http://dx.doi.org/10.1016/j.vaccine.2008.11.002.

Kikuta, A., F. Gardezi, V. Dubey, and A. Taddio. 2011. "Practices and Perceptions Regarding Pain and Pain Management during Routine Childhood Immunizations: Findings from a Focus-Group Study with Nurses Working at Toronto Public Health, Ontario." Canadian Journal of Infectious Diseases and Medical Microbiology 22 (2): 43-8. http://dx.doi.org/10.1155/2011/381864.

Kulig, J.C., C.J. Meyer, S.A. Hill, and C.E. Handley. 2002. "Refusals and Delay of Immunization within Southwest Alberta: Understanding Alternative Beliefs and Religious Perspectives." Canadian Journal of Public Health 93 (2): 109-12.

Loewenthal, K.M., and C. Bradley. 1996. "Immunization Uptake and Doctors' Perceptions of Uptake in a Minority Group: Implications for Interventions." Psychology Health and Medicine 1 (2): 223-30. http:// dx.doi.org/10.1080/13548509608400020.

Omer, B.S., A.D. Salmon, A.W. Orenstein, P. deHart, and N. Halsey. 2009. "Vaccine Refusal, Mandatory Immunization, and the Risks of Vaccine-Preventable Diseases." New England Journal of Medicine 360 (19): 1981-8. http://dx.doi.org/10.1056/nEJMsa0806477

Parvez, E., J. Stinson, H. Boon, J. Goldman, V. Shah, and A. Taddio. 2010. "Mothers' Beliefs About Analgesia during Childhood Immunization." Paediatrics \& Child Health 15: 289-93.

Poltorak, M., M. Leach, J. Fairhead, and J. Cassell. 2005. "'MMR talk' and Vaccination Choices: An Ethnographic Study in Brighton." Social Science \& Medicine 61 (3): 709-19. http://dx.doi.org/10.1016/j.socscimed.2004.12.014.

Salmon, D.A., S.P. Teret, C.R. Macintyre, D. Salisbury, M.A. Burgess, and N.A. Halsey. 2006. "Compulsory Vaccination and Conscientious or Philosophical Exemptions: Past, Present, and Future." The Lancet 367 (9508): 436-42. http://dx.doi.org/10.1016/ S0140-6736(06)68144-0.

Schwartz, J.L., and A.L. Caplan. 2011. "Ethics of Vaccination Programs." Current Issues in Virology 1: 263-7.

Siegal, G., N. Siegal, and R.J. Bonnie. 2009. "An Account of Collective Actions in Public Health." American Journal of Public Health 99 (9): 1583-7. http://dx.doi.org/10.2105/ AJPH.2008.152629.

Stern, A.M., and Markel, H. 2005. "The History of Vaccines and Immunization: Familiar Patterns, New Challenges." Health Affairs 24 (3): 611-21. http://dx.doi. org/10.1377/hlthaff.24.3.611.

Stockwell, M.S., M. Irigoyen, R.A. Martinez, and S. Findley. 2011. "How Parents' Negative Experiences at Immunization Visits Affect Child Immunization Status in a Community in New York City." Public Health Reports 126: 24-32. 
Taddio, A., M. Appleton, R. Bortolussi, C. Chambers, V. Dubey, S. Halperin, A. Hanrahan, M. Ipp, D. Lockett, n. MacDonald, et al. 2010. "Reducing the Pain of Childhood Vaccination: An Evidence-Based Clinical Practice Guideline." Canadian Medical Association Journal 182 (18): 1989-95. http://dx.doi.org/10.1503/ cmaj.092048.

Taddio, A., C.T. Chambers, S.A. Halperin, M. Ipp, D. Lockett, M.J. Rieder, and V. Shah. 2009. "Inadequate Pain Management during Routine Childhood Immunizations: The Nerve of It." Clinical Therapeutics 31 (Suppl B): S152-67. http://dx.doi.org/10.1016/j.clinthera.2009.07.022.

Taddio, A., M. Ipp, S. Thivakaran, A. Jamal, C. Parikh, J. Smart, J. Sovran, D. Stephens, and J. Katz. 2012. "Survey of the Prevalence of Immunization Non-Compliance Due to Needle Fears in Children and Adults." Vaccine 30 (32): 4807-12. http://dx.doi. org/10.1016/j.vaccine.2012.05.011.

Tarrant, M., and D. Gregory. 2001. "Mothers Perceptions of Childhood Immunizations in First Nations Communities of the Sioux Lookout Zone." Canadian Journal of Public Health 92 (1): 42-5.

- 2003. "Exploring Childhood Immunization Uptake with First Nations Mothers in North-Western Ontario, Canada." Journal of Advanced Nursing 41 (1): 63-72. http:// dx.doi.org/10.1046/j.1365-2648.2003.02507.x.

Ulmer, J.B., U. Valley, and R. Rappuoli. 2006. "Vaccine Manufacturing: Challenges and Solutions." Nature Biotechnology 24 (11): 1377-83. http://dx.doi.org/10.1038/ nbt1261.

Verweij, M.M., and A.A. Dawson. 2004. "Ethical Principles for Collective Immunisation Programmes." Vaccine 22 (23-4): 3122-6. http://dx.doi.org/10.1016/j. vaccine.2004.01.062.

"What is Christian Science?" 2011. Christian Science. Accessed 2 May 2014, http://christianscience.com/ what-is-christian-science.

Wolfe, M.R., and K.L. Sharp. 2002. "Anti-Vaccinationists Past and Present." BMJ: British Medical Journal 325 (7361): 430-2.

Woo, E.J., R. Ball, A. Bostrom, S. Shadomy, L.K. Ball, G. Evans, and M. Braun. 2004. "Vaccine Risk Perception among Reporters of Autism after Vaccination: Vaccine Adverse Event Reporting System 1990-2001." American Journal of Public Health 94 (6): 990-5. http://dx.doi.org/10.2105/AJPH.94.6.990.

Wynne, B. 2006. "Public Engagement as a Means of Restoring Public Trust in Science - Hitting the Notes, but Missing the Music?" Community Genetics 9 (3): 211-20. 